\title{
HETEROSIS AND COMBINING ABILITY ANALYSIS FOR YIELD AND ITS COMPONENTS IN DURUM WHEAT( TRITICUM DURUM, DESF.).
}

\author{
Salah El-Din Ahmed Abd- El-Majeed \\ National Wheat Research Program, Field Crops Research Institute \\ Agricultural Research Center, Giza.
}

\begin{abstract}
Analysis of variance for combining ability in 6-parent diallel (excluding reciprocals) of durum wheat (Triticum durum, Desf.) revealed the following results. Desirable heterosis values over better parent were observed for some cross combinations for all the studied traits; plant height, days to heading, days to maturity, No. spikes / plant, No. kernels / spike, 1000 kernel weight and grain yield / plant. The analysis of variance for combining ability showed that mean squares due to general combining ability (GCA) as well as specific combining ability (SCA) were highly significant for all studied traits indicating the importance of both additive and non-additive gene effects with the preponderance of non-additive type of gene effects for yield and most of its components.
\end{abstract}

\section{Key Words: Heterosis, General combining ability and Specific combining ability.}

\section{INTRODUCTION}

Wheat today occupies a very great position among cereals in Egypt. Recent breakthrough in wheat production is not sufficient to meet the rapidly growing population of the country which is estimated to cross one hundred million by the next couple of decades. This necessities the acceleration of improvement in this crop. For this, combining ability studies are frequently used by plant breeders to evaluate newly developed cultivars for their parental usefulness and to assess the gene action involved in various characters, so as to design an efficient breeding plan for further genetic upgrading of the existing material (Menon Uma Ans Sharma (1994).

Heterosis in the $F_{1}$ progeny has been used as an indicator of genetic diversity between parents. Magnitude and direction of heterosis for mid- and better parent are very important in the exploitation of heterosis. Hence, the present study was conducted to estimate the heterosis values for both mid and better parent, general (GCA) and specific(SCA) combining ability and correlation coefficient between all pairs of the studied traits.

\section{MATERIALS AND METHODS}

Six varieties and/or liens of durum wheat (Triticum durum, Desf.) namely Bani-suief $3\left(\mathrm{P}_{1}\right)$, Brachoua /3/ win / kif 's' // Ruff's'/Fg 's' $\left(\mathrm{P}_{2}\right)$, Kucuk $\left(\mathrm{P}_{3}\right)$, SRN1/Laru /3/Yav1 /FGO//Rch/4/Lican $\left(\mathrm{P}_{4}\right)$, ZEGZAG/ ALTAR84 // Dipper2 $\left(\mathrm{P}_{5}\right)$ and Zeina-3 $\left(\mathrm{P}_{6}\right)$.

The present study was carried out at Sids Agricultural Research Station during the two successive seasons 2001/2002 and 2002/2003. In the first season, the parents were crossed in all possible combinations excluding reciprocals using hand emasculation and pollination to produce the hybrid seeds of $15 \mathrm{~F}_{1}$ 's .In the

Fayoum J. Agric. Res. \& Dev., Vol.19, No.1, January, 2005 
second season, the resulting $15 \mathrm{~F}_{1}$ 's along with the six parents were grown in a randomized complete block design with three replications. Each of the parents and their $F_{1}$ 's were represented by two rows $10 \mathrm{~cm}$ between seeds and $30 \mathrm{~cm}$ between rows with 3 meter long. All recommended agronomic practices were applied. Twenty competitive plants were selected randomly for recording observations on plant height (PLH), days to heading (DHE), days to maturity (DMA), number of spikes/plant (S/P), number of kernels/spike (K/S), 1000 kernel weight $(\mathrm{KW})$ and grain yield/plant $(\mathrm{GY} / \mathrm{P})$.

Collected data were subjected to the usual analysis followed for a randomized complete block design proposed by Snedecor and Cochran, 1980 using computer soft ware MSTATC program. Heterosis was computed according to Bhatt (1973). The combining ability analysis was done according to Method II, Model 1 of Griffing (1956).

\section{RESULTS AND DISCUSSION}

\section{Mean performance :}

Significant differences were observed among the parental genotypes as well as their $F_{1}$ 's for all studied traits. The variation due to parents vs. crosses were highly significant for all studied traits. (Table 1).

Among the parental genotypes, $\mathrm{P}_{5}$ possessed the highest values of days to heading, days to maturity, number of spikes/plant and grain yield/plant, whereas it was the shortest parent for plant height .On the other hand, $\mathrm{P}_{1}$ gave the lowest values of days to heading, days to maturity, 1000 -kernels weight and grain yield/plant. The $\mathrm{P}_{3}, \mathrm{P}_{6}$, and $\mathrm{P}_{2}$ gave the highest value of plant height, number of kernels /spike and 1000- kernels weight, respectively.

With respect to $\mathrm{F}_{1}$ 's, data obtained indicated that the cross combination $\mathrm{P}_{1} \times \mathrm{P}_{2}$ was the tallest cross while $\mathrm{P}_{1} \times \mathrm{P}_{4}$ showed the lowest plant height. For days to heading $\mathrm{P}_{1} \times \mathrm{P}_{3}$ was the earliest in spike emergence, while the $\mathrm{P}_{4} \times \mathrm{P}_{5}$ was the latest in spike emergence (Table1).

Concerning days to maturity the cross $\mathrm{P}_{1} \times \mathrm{P}_{3}$ was the earliest for days to maturity, while $\mathrm{P}_{4} \times \mathrm{P}_{5}$ was the latest. With regard to number of spikes/ plant the crosses $\mathrm{P}_{1} \times \mathrm{P}_{4}$ and $\mathrm{P}_{2} \times \mathrm{P}_{6}$ gave the height values while the cross $\mathrm{P}_{5} \times \mathrm{P}_{6}$ gave lowest value. For kernels/spike cross $\mathrm{P}_{3} \times \mathrm{P}_{5}$ showed the highest value, while the cross $\mathrm{p}_{2} \mathrm{x} \mathrm{p}_{6}$ produced the lowest value. Cross combination $\mathrm{P}_{2} \times \mathrm{P}_{3}$ produced the heaviest 1000- kernels weight, while the cross $\mathrm{P}_{4} \times \mathrm{P}_{6}$ produced the lightest 1000- kernels weight. For grain yield, it was observed that the cross $\mathrm{P}_{3} \times \mathrm{P}_{5}$ produced the highest grain yield/plant, whereas, the cross $\mathrm{P}_{3} \times \mathrm{P}_{4}$ gave the lowest value.

\section{Heterosis effect :}

Desirable heterosis values over better parent were observed for plant height in the crosses $\mathrm{P}_{1} \times \mathrm{P}_{2}, \mathrm{P}_{2} \times \mathrm{P}_{4}, \mathrm{P}_{2} \times \mathrm{P}_{5}$ and $\mathrm{P}_{4} \times \mathrm{P}_{6}$. For days to heading (negative direction) in the crosses $\mathrm{P}_{1} \times \mathrm{P}_{3}, \mathrm{P}_{1} \times \mathrm{P}_{4}$ and $\mathrm{P}_{1} \times \mathrm{P}_{6}$ showed heterosis relative to mid-parent. For number of spikes/plant, most of the crosses under study possessed highly significant heterosis over better parent. For number of kernels/spike, only five crosses showed significant heterosis over better parent $\left(\mathrm{P}_{2} \times \mathrm{P}_{3}, \mathrm{P}_{3} \times \mathrm{P}_{4}, \mathrm{P}_{3} \times \mathrm{P}_{5}, \mathrm{P}_{3} \times \mathrm{P}_{6}\right.$ and $\left.\mathrm{P}_{5} \times \mathrm{P}_{6}\right)$, For 1000-kernel weight, highly significant heterosis over better parent were observed in four crosses $\left(\mathrm{P}_{1} \times \mathrm{P}_{3}, \mathrm{P}_{1}\right.$ x $\mathrm{P}_{5}, \mathrm{P}_{1} \times \mathrm{P}_{6}, \mathrm{P}_{3} \times \mathrm{p}_{5}$ and $\mathrm{P}_{3} \times \mathrm{P}_{6}$ ). For grain yield per plant, most of the crosses showed heterosis values over better parent (Table 2).

Fayoum J. Agric. Res. \& Dev., Vol.19, No.1, January, 2005 
HETEROSIS AND COMBINING ABILITY ANALYSIS FOR

Table (1): Mean performance of the studied six parents and their $F_{1}$ 's for plant height (PLH), days to heading (DHE), days to maturity (DMA), number of spikes/plant (S/P), number of kernels/spike (K/S), 1000-kernel weight (KW) and grain yield /plant( GY/P).

\begin{tabular}{|c|c|c|c|c|c|c|c|}
\hline PARENTS & PLH & DHE & DMA & $\mathbf{S} / \mathbf{P}$ & $\mathbf{K} / \mathbf{S}$ & KW & GY/P \\
\hline $\mathbf{P}_{1}$ & 102.33 & 109.33 & 156.00 & 15.0 & 75.00 & 51.40 & 37.70 \\
\hline $\mathbf{P}_{2}$ & 105.00 & 105.00 & 153.00 & 13.00 & 60.33 & 64.65 & 43.22 \\
\hline $\mathbf{P}_{3}$ & 106.33 & 106.00 & 155.33 & 11.67 & 61.00 & 54.55 & 51.00 \\
\hline $\mathbf{P}_{4}$ & 104.67 & 107.67 & 156.33 & 15.67 & 67.33 & 54.16 & 43.07 \\
\hline$P_{5}$ & 93.43 & 103.00 & 155.33 & 15.67 & 75.67 & 55.31 & 61.67 \\
\hline $\mathbf{P}_{6}$ & 105.67 & 106.67 & 155.33 & 12.67 & 69.33 & 54.50 & 42.44 \\
\hline \multicolumn{8}{|l|}{ crosses } \\
\hline $\mathbf{P}_{1} \mathbf{X} \mathbf{P}_{2}$ & 109.33 & 107.67 & 156.33 & 13.00 & 73.00 & 64.21 & 38.43 \\
\hline $\mathbf{P}_{1} \mathbf{X} \mathbf{P}_{3}$ & 104.00 & 104.67 & 155.33 & 17.00 & 72.67 & 61.31 & 59.77 \\
\hline$P_{1} \times P_{4}$ & 97.67 & 106.00 & 157.00 & 18.33 & 73.00 & 54.98 & 53.08 \\
\hline $\mathbf{P}_{1} \mathbf{X} \mathbf{P}_{5}$ & 100.33 & 108.67 & 156.33 & 17.33 & 66.33 & 60.30 & 56.33 \\
\hline $\mathbf{P}_{1} \mathbf{X} \mathbf{P}_{6}$ & 102.67 & 106.00 & 159.00 & 17.00 & 75.33 & 58.41 & 31.19 \\
\hline $\mathbf{P}_{2} \mathbf{X} \mathbf{P}_{3}$ & 104.67 & 105.67 & 159.67 & 11.67 & 64.67 & 67.74 & 43.12 \\
\hline $\mathbf{P}_{2} \mathbf{X} \mathbf{P}_{4}$ & 108.00 & 108.67 & 158.00 & 15.67 & 64.00 & 62.56 & 62.53 \\
\hline $\mathbf{P}_{2} \mathbf{X} \mathbf{P}_{5}$ & 108.00 & 107.33 & 157.33 & 17.33 & 60.33 & 66.05 & 57.72 \\
\hline $\mathbf{P}_{2} \mathbf{X} \mathbf{P}_{6}$ & 106.67 & 109.00 & 157.67 & 18.33 & 59.33 & 60.63 & 72.71 \\
\hline $\mathbf{P}_{3} \mathbf{X} \mathbf{P}_{4}$ & 105.33 & 106.00 & 158.33 & 17.00 & 70.00 & 52.59 & 30.26 \\
\hline $\mathbf{P}_{3} \mathbf{X} \mathbf{P}_{5}$ & 102.00 & 107.33 & 158.67 & 14.67 & 81.00 & 59.95 & 74.00 \\
\hline $\mathbf{P}_{3} \mathbf{X} \mathbf{P}_{6}$ & 100.33 & 107.67 & 157.67 & 13.33 & 73.33 & 58.81 & 40.54 \\
\hline $\mathbf{P}_{4} \mathbf{X} \mathbf{P}_{5}$ & 102.57 & 110.00 & 160.00 & 13.00 & 73.00 & 58.05 & 42.21 \\
\hline $\mathbf{P}_{4} \mathbf{X} \mathbf{P}_{6}$ & 108.00 & 106.67 & 159.33 & 17.33 & 68.33 & 43.59 & 46.42 \\
\hline$P_{5} \times P_{6}$ & 103.00 & 107.33 & 159.67 & 11.33 & 80.00 & 55.53 & 40.58 \\
\hline LSD .05 & 3.025 & 2.489 & 3.164 & 2.899 & 5.656 & 6.404 & 17.74 \\
\hline .01 & 4.040 & 3.327 & 4.229 & 3.875 & 7.561 & 8.559 & 23.35 \\
\hline
\end{tabular}

Analysis of variance :

The analysis of variance for combining ability showed that mean squares due to GCA as well as SCA were highly significant for all studied traits, indicating the important role of both additive and non additive variances in the inheritance of the studied traits. GCA/SCA values were more than unity in plant height, number of kernels per spike and 1000-kernel weight indicating that the additive variance was more important than the dominance ones in inheritance of these traits. (Table 3).

Grain yield/plant had the higher GCA variance (125.68) followed by number of kernels/spike (82.07). Number of kernels per spike had the greatest GCA/SCA (4.74) followed by number of kernels/spike (3.01). The present obtained results were in harmony with those obtained by Verma and Luthra (1983), Afiah (1999), Yadav and Nasinghani (2000), Ashoush et al, (2001), Mahmoud (2002) and Salgotra et al, (2002).

Fayoum J. Agric. Res. \& Dev., Vol.19, No.1, January, 2005 
General combining ability effects :

The estimates of general combining effects are presented in Table (4). $\mathrm{P}_{2}$ was found to be good combiner for plant height (2.528), P1 and $\mathrm{P}_{5}$ were seemed to be good combiners for number of kernels per spike (2.833 and 3.042, respectively), $\mathrm{P}_{2}$ was found to be good combiner for 1000-kenel weight (5.509) and $\mathrm{P}_{2}$ and $\mathrm{P}_{5}$ were good combiners for grain yield per plant.

Table (2): Heterosis (H) values \% for mid-parent (MP) and bitter-parent (BP) for all characters studied of F1 diallel.

\begin{tabular}{|c|c|c|c|c|c|c|c|c|}
\hline Crosses & $\mathbf{H}$ & PLH & DHE & DMA & $\mathbf{S} / \mathbf{P}$ & $\mathbf{K} / \mathbf{S}$ & KW & GY/P \\
\hline \multirow[t]{2}{*}{$\mathbf{P}_{1} \times \mathbf{P}_{2}$} & MP & $5.466 * *$ & 0.467 & 1.187 & $-7.140 * *$ & $7.880 * *$ & $10.660 * *$ & -5.010 \\
\hline & $\mathrm{BP}$ & $4.124 * *$ & $2.543^{*}$ & 2.176 & $-13.330 * *$ & -2.670 & -0.681 & -11.100 \\
\hline \multirow[t]{2}{*}{$\mathbf{P}_{1} \mathbf{X} \mathbf{P}_{3}$} & MP & -0.316 & $-2.790 *$ & -0.214 & $27.500 * *$ & $6.863 * *$ & $15.730 * *$ & $34.770 * *$ \\
\hline & BP & -2.191 & -0014 & -0.429 & $13.330 * *$ & $-3.107 * *$ & 12.391 & $17.200 * *$ \\
\hline \multirow[t]{2}{*}{$\mathbf{P}_{1} \times \mathbf{P}_{4}$} & MP & $-5.630 * *$ & $-2.300^{*}$ & 0.534 & $19.570 * *$ & 2.576 & 4.168 & $31.438 * *$ \\
\hline & $\mathrm{BP}$ & $-6.68 * *$ & -1.551 & 1.075 & $16.980 * *$ & -2.667 & 1.514 & $24.845 * *$ \\
\hline \multirow[t]{2}{*}{$\mathbf{P}_{1} \times \mathbf{P}_{5}$} & MP & 2.503 & $2.355^{*}$ & -0.422 & $13.04 * *$ & $-11.95^{* *}$ & $13.013 * *$ & 13.385 \\
\hline & $\mathrm{BP}$ & -1.954 & $5.505 * *$ & -0.212 & $10.590 * *$ & $-12.340 * *$ & $9.022 * *$ & -8.654 \\
\hline \multirow[t]{2}{*}{$\mathbf{P}_{1} \times \mathbf{P}_{6}$} & MP & -1.282 & -1.851 & 2.141 & $22.89 * *$ & 4.388 & $10.305 * *$ & $-22.150 * *$ \\
\hline & BP & -2.839 & -0.628 & 2.363 & $8.557 * *$ & 0.440 & 7.174* & $-26.500 * *$ \\
\hline \multirow[t]{2}{*}{$\mathbf{P}_{\mathbf{2}} \mathbf{X} \mathbf{P}_{3}$} & MP & -0.946 & 0.158 & $3.568 *$ & $-5.410 * *$ & $6.593 * *$ & $13.658 * *$ & -8.472 \\
\hline & $\mathrm{BP}$ & -1.561 & 0.638 & $4.359 * *$ & $-10.230 * *$ & $6.016 *$ & 4.780 & 15.451 \\
\hline \multirow[t]{2}{*}{$\mathbf{P}_{2} \times \mathbf{P}_{4}$} & MP & $3.020 *$ & 2.194 & 2.155 & $9.300 * *$ & 0.261 & 5.308 & $44.940 * *$ \\
\hline & $\mathrm{BP}$ & 2.857 & $3.495 * *$ & $3.268 *$ & 0.000 & -4.946 & -3.233 & $45.200 * *$ \\
\hline \multirow[t]{2}{*}{$\mathbf{P}_{2} \times \mathbf{P}_{5}$} & MP & $9.190 * *$ & $3.205 * *$ & 2.050 & $20.930 * *$ & $-11.280 * *$ & $10.119 * *$ & 10.048 \\
\hline & $\mathrm{BP}$ & $3.170 *$ & $4.204 * *$ & 1.288 & $10.590 * *$ & $-20.270 * *$ & 2.166 & -6.410 \\
\hline \multirow[t]{2}{*}{$\mathbf{P}_{2} \mathbf{X} \mathbf{P}_{6}$} & MP & 1.266 & $2.998 * *$ & 2.270 & $42.860 * *$ & $-8.480 * *$ & 1.762 & $69.750 * *$ \\
\hline & $\mathrm{BP}$ & 0.946 & 3.810 & 3.052 & $41.000 * *$ & $-14.420 * *$ & -6.218 & $68.250 * *$ \\
\hline \multirow[t]{2}{*}{$\mathbf{P}_{\mathbf{3}} \mathbf{X} \mathbf{P}_{4}$} & MP & -0.158 & -0.780 & 1.604 & $24.930 * *$ & $9.091 * *$ & -3.250 & $-35.660 * *$ \\
\hline & BP & -0.941 & 0.000 & $3.219^{*}$ & $8.488 * *$ & $3.966 * *$ & -3.593 & $-40.660 * *$ \\
\hline \multirow[t]{2}{*}{$\mathbf{P}_{3} \times \mathbf{P}_{5}$} & MP & 2.119 & 2.711 & 2.146 & $7.317 * *$ & $18.540 * *$ & $9.133 * *$ & $31.360 * *$ \\
\hline & BP & $-4.070 * *$ & $4.204 * *$ & 2.150 & $-6.382 *$ & $7.044 * *$ & $8.389 *$ & $19.990 *$ \\
\hline \multirow[t]{2}{*}{$\mathbf{P}_{3} \times \mathbf{P}_{6}$} & MP & $-5.350 * *$ & 1.254 & 1.502 & $9.589 * *$ & $12.53 * *$ & $7.868 * *$ & -13.240 \\
\hline & $\mathrm{BP}$ & $-5.640 * *$ & 1.575 & 1.506 & $5.209 *$ & $5.770 *$ & $7.809 *$ & $-20.520 *$ \\
\hline \multirow[t]{2}{*}{$\mathbf{P}_{4} \times \mathbf{P}_{5}$} & MP & $3.550 * *$ & $4.430 * *$ & 2.150 & $-17.020 * *$ & 2.098 & $6.053 *$ & $-19.390 *$ \\
\hline & $\mathrm{BP}$ & -2.010 & $6.796 * *$ & 2.150 & $-17.040 * *$ & -3.528 & 4.954 & $-31.550 * *$ \\
\hline \multirow[t]{2}{*}{$\mathbf{P}_{4} \times \mathbf{P}_{6}$} & MP & $2.694 *$ & -0.467 & 2.246 & $22.350 * *$ & 0.000 & $-19.770 * *$ & 8.584 \\
\hline & BP & 2.205 & 0.000 & 2.575 & $10.590 * *$ & -1.442 & $-20.020 * *$ & 7.328 \\
\hline \multirow[t]{2}{*}{$\mathbf{P}_{5} \mathbf{X} \mathbf{P}_{6}$} & MP & $3.466^{*}$ & $2.385^{*}$ & $2.790 *$ & $-20.000 * *$ & $10.350 * *$ & 1.141 & $-22.050 * *$ \\
\hline & $\mathrm{BP}$ & -2.527 & $4.204 * *$ & 2.790 & $-27.690 * *$ & $5.720 *$ & 0.398 & $-34.200 * *$ \\
\hline \multirow{2}{*}{$\begin{array}{l}\text { LSD } \\
0.05\end{array}$} & MP & 2.619 & 2.156 & 2.740 & 2.511 & 4.899 & 5.546 & 15.131 \\
\hline & $\mathrm{BP}$ & 3.025 & 2.461 & 3.164 & 5.197 & 5.656 & 6.404 & 17.472 \\
\hline \multirow{2}{*}{$\begin{array}{c}\text { LSD } \\
0.01\end{array}$} & MP & 3.501 & 2.882 & 4.968 & 3.356 & 6.548 & 7.413 & 20.225 \\
\hline & $\mathrm{BP}$ & 4.043 & 3.290 & 4.229 & 6.946 & 7.561 & 8.559 & 23.353 \\
\hline
\end{tabular}

* Significant at P>0.05. ** Significant at $\mathrm{P}>0.01$.

Fayoum J. Agric. Res. \& Dev., Vol.19, No.1, January, 2005 
Table (3): Observed mean squares from ordinary and combining ability analysis in ${ }_{\mathrm{F} 1}$ 's diallel.

\begin{tabular}{|c|c|c|c|c|c|c|c|c|}
\hline S.O.V & DF & PLH & DHE & DMA & S/P & K/S & KW & GY/P \\
\hline Genotypes & 20 & $43.18^{* *}$ & $8.56^{* *}$ & $10.14^{* *}$ & $16.18^{* *}$ & $122.9^{* *}$ & $94.95^{* *}$ & $456.9^{* *}$ \\
\hline Parents & 5 & $70.15^{* *}$ & $14.32^{* *}$ & $4.09^{* *}$ & $8.86^{* *}$ & $130.62^{* *}$ & $62.35^{* *}$ & $220.13^{* *}$ \\
\hline Crosses & 14 & $35.11^{* *}$ & $6.26^{* *}$ & $5.83^{* *}$ & $17.76^{* *}$ & $124.57^{* *}$ & $103.87^{* *}$ & $563.42^{* *}$ \\
\hline P vs. C & 1 & 21.32 & 12.01 & 100.8 & 30.67 & 60.98 & 133.14 & 149.53 \\
\hline GCA & 5 & $27.12^{* *}$ & $1.48^{* *}$ & $2.27^{* *}$ & $4.88^{* *}$ & $82.07^{* *}$ & $77.52^{* *}$ & $125.68^{* *}$ \\
\hline SCA & 15 & $10.15^{* *}$ & $3.31^{* *}$ & $3.75^{* *}$ & $5.56^{* *}$ & $27.26^{* *}$ & $16.36^{* *}$ & $161.18^{* *}$ \\
\hline Error & 40 & 3.36 & 2.28 & 3.68 & 3.09 & 11.76 & 15.08 & 112.22 \\
\hline GCA/SCA & & 2.67 & 0.45 & 0.61 & 0.88 & 3.01 & 4.74 & 0.78 \\
\hline
\end{tabular}

Table (4): Estimates of general combining ability effects of parents of the studied traits in the $F_{1}$ diallel.

\begin{tabular}{|c|c|c|c|c|c|c|c|}
\hline Parents & PLH & DHE & DMA & S/P & K/S & KW & GY/P \\
\hline $\mathbf{P}_{\mathbf{1}}$ & $-1.01^{* *}$ & 0.361 & -0.569 & $0.917^{* *}$ & $2.833^{* *}$ & -0.552 & -3.558 \\
\hline $\mathbf{P}_{\mathbf{2}}$ & $2.528^{* *}$ & -0.056 & -0.694 & -0.417 & $-5.71^{* *}$ & $5.509^{* *}$ & 2.287 \\
\hline $\mathbf{P}_{\mathbf{3}}$ & 0.278 & $-0.681^{*}$ & -0.028 & -1.042 & -0.50 & 0.383 & 0.787 \\
\hline $\mathbf{P}_{\mathbf{4}}$ & 0.515 & 0.486 & 0.597 & $0.917^{* *}$ & -0.583 & $-3.29^{* *}$ & -2.753 \\
\hline $\mathbf{P}_{\mathbf{5}}$ & $-2.96^{* *}$ & -0.264 & 0.264 & -0.042 & $3.042^{* *}$ & 0.511 & $6.439^{* *}$ \\
\hline $\mathbf{P}_{\mathbf{6}}$ & 0.653 & 0.153 & 0.431 & -0.333 & 0.917 & -2.56 & -3.293 \\
\hline LSD gi 0.05 & 0.691 & 0.577 & 0.721 & 0.663 & 1.291 & 1.460 & 3.987 \\
\hline gi 0.01 & 0.923 & 0.759 & 0.964 & 0.886 & 1.725 & 1.952 & 5.330 \\
\hline gi-gj 0.05 & 1.069 & 0.881 & 1.119 & 1.241 & 1.999 & 2.264 & 6.177 \\
\hline
\end{tabular}

\section{Specific combining ability :}

The estimates of SCA effects of all cross combinations are presented in (Table 5). The best cross combinations on the basis of SCA for plant height were $\mathrm{P}_{1} \times \mathrm{P}_{2}$ (3.994), $\mathrm{P}_{2} \times \mathrm{P}_{5}$ (4.940) and $\mathrm{P}_{4} \times \mathrm{P}_{6}$ (3.007). For days to heading, three cross combinations were good for SCA (negative direction toward earliness) $\mathrm{P}_{1}$ $\times \mathrm{P}_{3}, \mathrm{P}_{1} \times \mathrm{P}_{4}$ and $\mathrm{P}_{1} \times \mathrm{P}_{6}$. None of the cross combinations seemed to be good on the basis of SCA effects for days to maturity (negative direction toward earliness) $\mathrm{P}_{1} \times \mathrm{P}_{3}, \mathrm{P}_{1} \times \mathrm{P}_{4}$ and $\mathrm{P}_{1} \times \mathrm{P}_{5}$. Four cross combinations gave good values for SCA in comparing to the cross for number of spikes per plant, $\mathrm{P}_{1} \times \mathrm{P}_{3}$ (2.077), $\mathrm{P}_{2} \times \mathrm{P}_{5}$ (2.744), $\mathrm{P}_{2} \times \mathrm{P}_{6}$ (4.036) and $\mathrm{P}_{3} \times \mathrm{P}_{4}$ (2.077). For number of kernels per spike, three cross combinations were the best on the basis of SCA effect, $\mathrm{P}_{1} \times \mathrm{P}_{2}$ (6.208), $\mathrm{P}_{3} \times \mathrm{P}_{5}$ (8.792) and $\mathrm{P}_{5} \times \mathrm{P}_{6}(6.375)$.

None of crosses showed good SCA effects for 1000-kernel weight. For grain yield per plant, four cross combinations were very good for SCA effects, $\mathrm{P}_{1}$ $\times \mathrm{P}_{3}$ (13.49), $\mathrm{P}_{2} \times \mathrm{P}_{4}$ (14.04), $\mathrm{P}_{2} \times \mathrm{P}_{6}$ (24.762) and $\mathrm{P}_{3} \times \mathrm{P}_{5}$ (17.73). These data were partially similar to those obtained by Verma and Luthra 1983, Hassan, (1997), El-Beially and El-Sayed, (2002).

Fayoum J. Agric. Res. \& Dev., Vol.19, No.1, January, 2005 
Table (5): Estimates of specific combining ability for crosses studied in the $F_{1}$ diallel.

\begin{tabular}{|c|c|c|c|c|c|c|c|}
\hline CROSSES & PLH & DHE & DMA & $\mathbf{S} / \mathbf{P}$ & $\mathbf{K} / \mathbf{S}$ & KW & GY/P \\
\hline $\mathbf{P}_{1} \mathbf{X ~} \mathbf{P}_{2}$ & $3.994 * *$ & 0.393 & 0.375 & $-2.55^{* *}$ & $6.21 * *$ & 1.196 & -9.24 \\
\hline $\mathbf{P}_{1} \times \mathbf{P}_{3}$ & 0.911 & $-1.982 *$ & -1.292 & $2.077^{*}$ & 0.667 & 3.416 & $13.49 *$ \\
\hline $\mathbf{P}_{1} \mathbf{X P}_{4}$ & $-5.66^{* *}$ & $-1.815^{*}$ & -0.250 & 1.452 & 1.083 & 0.764 & 10.43 \\
\hline $\mathbf{P}_{1} \mathbf{X} \mathbf{P}_{5}$ & 0.482 & $1.601 *$ & -0.583 & 1.411 & $-9.21 * *$ & 2.281 & 5.00 \\
\hline $\mathbf{P}_{1} \times \mathbf{P}_{6}$ & -0.798 & $-1.482 *$ & 1.917 & 1.369 & 1.917 & 3.456 & -10.9 \\
\hline $\mathbf{P}_{2} \mathbf{X} \mathbf{P}_{3}$ & $-1.964 *$ & -0.565 & $.176 * *$ & $-.1923^{*}$ & 1.208 & 3.687 & -8.99 \\
\hline $\mathbf{P}_{2} \mathbf{X} \mathbf{P}_{4}$ & $1.132 *$ & 1.268 & 0.875 & 0.119 & 0.625 & 2.283 & 14.04* \\
\hline $\mathbf{P}_{2} \mathbf{X} \mathbf{P}_{5}$ & $4.940 *$ & 0.685 & 0.542 & $2.744 * *$ & $-6.67 * *$ & 1.973 & 0.03 \\
\hline $\mathbf{P}_{2} \times \mathbf{P}_{6}$ & -0.339 & $1.935^{*}$ & 0.780 & $4.036^{* *}$ & $-5.54 * *$ & -0.386 & $24.762 * *$ \\
\hline $\mathbf{P}_{3} \mathbf{X} \mathbf{P}_{4}$ & 0.715 & -.774 & 0.542 & $2.077 *$ & 1.417 & -2564 & -16.81 \\
\hline $\mathbf{P}_{3} \times \mathbf{P}_{5}$ & 0.557 & 1.310 & 1.280 & 0.702 & $8.792 * *$ & 0.993 & $17.730 * *$ \\
\hline $\mathbf{P}_{3} \times \mathbf{P}_{6}$ & $-4.42 * *$ & 1.226 & 0.042 & -0.339 & 3.250 & 2.927 & -6.00 \\
\hline $\mathbf{P}_{4} \times \mathbf{P}_{5}$ & 1.186 & $2.810^{* *}$ & 1.917 & $-2.92 * *$ & 0.875 & 2.771 & -10.4 \\
\hline $\mathbf{P}_{4} \times \mathbf{P}_{6}$ & $3.007 * *$ & -0.940 & 1.083 & 1.702 & -1.767 & $-8.624 * *$ & 3.51 \\
\hline $\mathbf{P}_{5} \times \mathbf{P}_{6}$ & 1.482 & 0.476 & 1.750 & $-3.34 * *$ & $6.375^{* *}$ & -.0481 & -11.52 \\
\hline \multicolumn{8}{|l|}{ LSD } \\
\hline Sij 0.05 & 1.897 & 1.559 & 1.984 & 1.818 & 3.545 & 4.013 & 10.95 \\
\hline 0.01 & 2.535 & 2.084 & 2.651 & 2.430 & 4.739 & 5.365 & 14.63 \\
\hline Sij-sik 0.05 & 2.830 & 2.329 & 2.959 & 2.713 & 5.290 & 5.989 & 16.34 \\
\hline 0.01 & 3.783 & 3.113 & 3.956 & 3.626 & 7.071 & 8.006 & 21.84 \\
\hline Sij-ski 0.05 & 2.620 & 2.155 & 2.757 & 2.511 & 4.899 & 5.545 & 15.13 \\
\hline 0.01 & 3.502 & 2.881 & 3.356 & 3.356 & 6.548 & 7.412 & 20.22 \\
\hline
\end{tabular}

\section{REFERENCES}

Afiah, S.A.N. (1999). Combining ability, association and path coefficient analysis of some wheat ( $T$. aestivum, L.) diallel crosses under desert conditions. J. Agric. Sci. Mansoura Univ., 24 (4): 1583-1596,1999.

Ashoush, H.A.; A.A. Hamada, and I.H. Darwish (2001). Heterosis and combining ability in F1 Diallel crosses of wheat (T.aestivum, L.) J. Agric. Sci. Mansoura Univ., 26 (5): 2579-2592.

Bhatt, G. M. (1973). Significance of path coefficient analysis in determining the nature of character association. Euphytica, 22:338-343.

El-Beially, I.E.M. and E.A.M. El-Sayed (2002). Heterosis and combining ability for some bread wheat crosses. J. Agric. Sci .Mansoura Univ., 27(9) : 5735- 5744.

Griffing, B. (1956). Concept of general and specific combining ability in relation to diallel crossing system. Aust. J. Biol. Res. 1: 23-26.

Hassan, E.E. (1997). Combining ability and factor analysis in durum wheat ( $T$. turgidum ). Zagazig J. Agric. Res. 24 (1): 23-36.

Mahmoud, A.S.A. (2002). Analysis of variance and its components of some hexaploid wheat crosses. M. Sc. Thesis, Agronomy Dept., Fac. Agric., Minia Univ. Egypt.

Menon Uma and S.N. Sharma (1994). Combining ability analysis for yield and its components in bread wheat over environments. Wheat information service. December 79: 18-23.

Fayoum J. Agric. Res. \& Dev., Vol.19, No.1, January, 2005 
Salgotra, R.K., K.S. Thakur, G.S. Sethi and J.K. Sharma (2002). Heterosis in winter x spring wheat crosses. Indian J. Genetics., 62(2) :104-106.

Snedecor, G.W. and W.G. Cochran (1980). Statistical Methods $7^{\text {th }}$ Ed. Iowa state Univ. Press, Ames, Iowa, U.S.A.

Verma, P.K. and O.P.Luthra (1983). Heterosis and combining ability analysis for yield and its attributes in Macaroni wheat. Indian J. Agric. Sci. 53 (6): 385-389.

Yadav, R.K. and V.G. Narsinghani (2000). Heterosis and inbreeding depression in wheat (T. aestivum L. and T. durum, Dest.) Indian J. Genet; 60 (3): 381- 382.

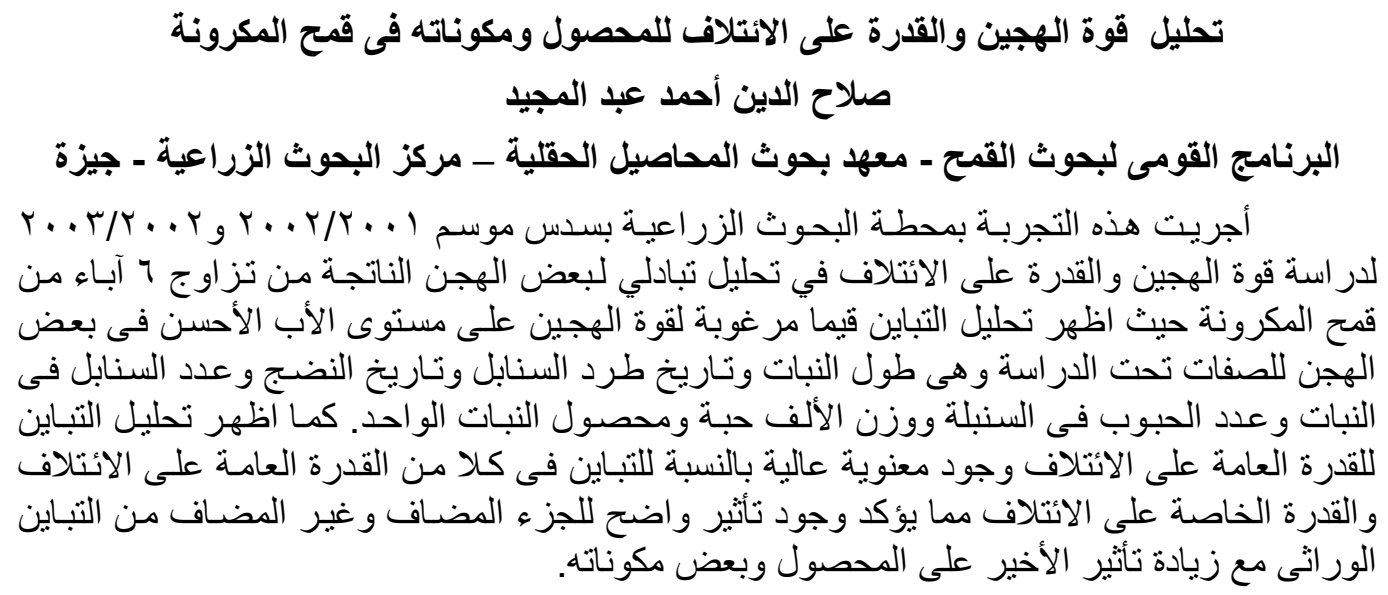

Fayoum J. Agric. Res. \& Dev., Vol.19, No.1, January, 2005 